\title{
Nutrition and dietetic-led gastrostomy pathway of care for insertion and removal contributes to outcomes
}

\author{
B. P. Talwar ${ }^{1}$ and R. Hewitt ${ }^{2}$ \\ Department of Nutrition \& Dietetics ${ }^{1}$, Department of Head \& Neck Cancer Services ${ }^{2}$, Department of Gastroenterology, \\ Department of Radiology, University College Hospital, University College Hospitals NHS Foundation Trust, \\ London, United Kingdom
}

There is no published pathway of care for coordinating prophylactic gastrostomy insertion before treatment and for facilitating removal during rehabilitation to prevent tube dependency for patients with head and neck cancer. The objective was to review the experience of systematically appraising implementation of a care pathway to promote early dietetic intervention to improve nutritional status, maximise clinical effectiveness with assessment for suitability and method of insertion with dedicated gastroenterology and radiology expertise, minimise nutrition complications, implement post-treatment dietary rehabilitation to prevent tube dependency and demonstrate advantages of a dietitian-led service as a core role within the head and neck multidisciplinary team setting.

This is a comparative study of retrospective and prospective data for 100 patients referred for gastrostomy placement between 20052007. First limb analysis and outcomes of fifty patients were identified with a need for 'head and neck' dietitians (HND) to lead and develop a gastrostomy care pathway in November 2006. HND implemented a site-specific pathway to meet objectives. Postimplementation second limb analysis and outcomes of fifty patients was then compared with first limb data to evaluate and standardise pathway of care.

Comparisons were made between first and second limb data respectively. Demographics were similar for age (58:59, first stage: second stage), gender (male, 34:34; female, 16:16), tumour staging (T1, 5:4; T2, 7:9; T3, 11:10; T4, 24:23), and treatment intent (curative, 43:43; palliative, 7:7), but varied for tumour site (oral cavity, 17:13; oropharynx, 4:14; hypopharynx, 7:4; larynx, 10:11; nasopharynx, $5: 3$; other, $7: 5$ ).

First limb data confirmed no pre-placement dietetic counselling for thirteen patients (26\%), of which five (10\%) received no dietetic input whilst the tube was sited. No single team member provided a uniform approach to patient and carer counselling or education. No post-treatment protocol existed to facilitate nutrition rehabilitation or tube removal. Limited dietetic service provision reflected a negative BMI trend at key episodes in the patient pathway. Sixteen patients $(32 \%)$ were admitted to hospital during treatment for nutritional management. Twenty-one patients $(42 \%)$ experienced greater than $1 \mathrm{~d}$ hospital admission post placement due to late dietetic referral requiring immediate nutrition support, delayed patient education for tube care or complications. Six patients (12\%) were inadequately assessed for method of insertion and thirty-three $(66 \%)$ received no prophylactic antibiotics before placement.

Pre- and post-treatment dietetic clinics provided a closed loop for insertion and removal of feeding tubes. Gastroenterology and radiology expertise consolidated patient selection criteria, suitability for method of insertion and post-placement care. Second limb analysis data reflected that fifty patients $(100 \%)$ received gastrostomy counselling by the dietitian at pre-treatment, no hospital admission for nutritional management and ongoing monitoring in the post-treatment clinic for dietary rehabilitation facilitating tube removal. Increased dietetic service provision resulted in a positive BMI trend at key episodes in the patient pathway. Forty-six patients (92\%) had overnight stay post placement, one (2\%) extending to $3 \mathrm{~d}$ and one $(2 \%) 30 \mathrm{~d}$ due to complications. Ten patients $(20 \%)$ screened by gastroenterology prevented radiological placement in two (4\%). All patients received prophylactic antibiotics.

Specialist dietitians implemented timely nutrition support, gastrostomy counselling and rehabilitation in dietetic clinics, with effective patient education supporting the consent process and preventing tube dependency. The dietetic-led specialist care pathway improved screening and intervention, reduced complications and hospital admissions, resulting in cost savings, efficient prophylactic gastrostomy placement and evidence for the dietitian's role to promote functional and patient-centred outcomes. 